TM-1437

(SSC-N-283)

0102.000

\title{
Deflection Analysis for an SSC Dipole Magnet with Two External Supports
}

\author{
Thomas H. Nicol
}

Fermi National Accelerator Laboratory

P.O. Box 500, Batavia, IL 60510 USA

January 9, 1987 


\title{
Dofloction Analysis for an SSC Dipolo Magnot With Two Extornal Supports
}

\author{
Thomas H. Nicol \\ Fermi National Accelerator Laboratory
}

Introduction

SSC dipole magnets are presently supported at $f i v e$ mounting locations coincident with the internal cold mass supports. There is growing interest in reducing the number of external supports from five to two for reasons of simplified installation and alignment and as a cost reduction measure. This reports examines the placement of two external supports required to minimize the deflection of the cold mass assembly.

Assumptions

For all of the discussions which follow, it is assumed the the vacuum vessel and cold mass are 653.5 inches long ( $16.6 \mathrm{~m})$ and that the cold mass is supported by five support posts located at the center, $+/-136.25$ inches, and $+/-272.50$ inches from the center. The cold mass weight is assumed to be $16907 \mathrm{lb}$ and includes the skin, laminations, coil assembly, radiation shields, and the posts themselves. The vacuum ressel is 0.250 inches thick carbon steel with a weight density of $0.286 \mathrm{lb} / \mathrm{cubic}$ inch. Finally, the weight of bellows and piping in the interconnection area is assumed to be negligible. Further notation is shown in Figure 1.

\section{Analysis}

The interaction between the cold mass and vacuum vessel is sufficiently complex and contains enough subtleties to warrant a finite element analysis of the two systems. Even a fairly crude beam model will yield very good approximations to the required deflections. Figure 1 includes a schematic of the finite element model used in the analysis.

Of interest are the vertical deflections of the cold mass and vacuum vessel at several points along the 
assembly length when subjected to a vertical acceleration of $1 \mathrm{G}$ (self-weight). Examination of the effect of support spacing is simply a matter of constraining the boundary of the vacuum vessel at different points along its length.

Figure 2 shows a plot of the cold mass vertical deflection vs. position along the assembly for five different support positions. Figure 3 shows a similar plot for the vacuum vessel. If the optimum support position is defined as that which yields equal end and mid-span deflections of the cold mass, it is apparent from Figure 2 that none of the cases shown represent the optimum (although the +/-181.67 inches case is very close). One or two iterations around this point yields a good optimum support spacing at $+/-180.86$ inches.

Figure 4 shows a plot of the vertical deflections of both the cold mass and vacuum vessel when the supports are spaced at this optimized position. Two plots of cold mass deflection are presented. One is the raw deflection result. A second contains the same data after correcting for a 0.011 inch 'DC' offset from zero. Note that the vertical scale for this plot is expanded from the previous two figures. It is clear from this figure that the optimum support position has very nearly been realized. The resulting cold mass end and mid-span deflections are 0.042 and 0.041 inches respectively in the case of the raw data and 0.031 and 0.030 inches in the case of the corrected data. This data is duplicated in tabular form in Table 1 .

Defining a support spacing ratio as the ratio of the support spacing to the overall magnet length, we find that the optimum occurs at ls/lt equal to 0.5535 (refer to Figure 1 for notation). While this is a more convenient way to look at support position, one should be aware that the above ratio is slightly sensitive to changes in the magnet length, weight, and cold mass support spacing. It is interesting to note that the analytical solution for the optimum support spacing ratio of a simply supported beam subject to either self-weight or a uniformly distributed load is 0.554 .

Figure 5 shows a plot of the average vertical displacement for each of the five cases from Figs. 1 and 2 plus the optimized case from Figure 3 . It must be noted that the average cold mass deflection shown in Figure 5 includes the 0.011 inch 'DC' offset described above and shown in Figure 4. If this offset is eliminated (by 
shimming, for example) the computed average cold mass deflection can be reduced from 0.028 to 0.016 inches.

Figure 6 shows a plot of the RMS deflection along the length of both the cold mass and racuum ressels. The RMS deflection is defined as the square root of the sum of the squares of the differences between the deflections along the length of either assembly and the associated average deflection. It has been shown in an earlier analysis (see reference 1) that the minimum average and minimum RMS deflections occur at slighly different values of support spacing. The analysis presented here contains too few cases to make the difference apparent.

Finally, in order to fully evaluate the viablility of the two-support system, it helps to know the sensitivity of the minimum deflection to changes in the vacuum vessel wall thickness (all other parameters remaining fixed). Figure 7 shows a plot of the maximum deflections for the cold mass and vacuum vessel for a series of wall

thicknesses (the current thickness is 0.250 inches). All of the values plotted assume a support spacing ratio of 0.5535 .

\section{Conclusions}

Reference 2 states that the cold mass must be supported such that the maximum sag between supports is less than $0.5 \mathrm{~mm}(0.020$ inches) and that the maximum allowable construction and alignment errors must be less than $0.7 \mathrm{~mm}(0.028$ inches) for a total of $1.2 \mathrm{~mm}(0.048$ inches). Given a corrected average deflection of 0.016 inches (see above) it seems that the two support option is a viable alternative to the five supports presently being used (at least from the deflection standpoint).

What remains to be answered is whether the vacuum vessel and stiffening rings are capable of resisting the bending loads imposed at the post attachment points by lateral loads on the cold mass assembly. Some early work indicated that the support feet were necessary to transfer those loads to the supporting foundation, thus minimizing the stresses in the vacuum vessel material. It is probable that thicker stiffening rings will eliminate this concern.

From a cost standpoint, it is doubtful whether the change to two external supports will be of much impact. It is true that the number of feet is reduced by $60 \%$, but 
five penetrations and stiffeners are still required at the post locations. Unfortunately, the optimum support placements do not coincide with existing penetrations so two additional stiffeners will likely be required at the foot locations.

Overall the scheme has the potential to greatly ease the time and effort involved in magnet installation and alignment. For those reasons alone it seems a worthy change to the present configuration.

\section{Roforoncos}

1. D.E. Groom, "Can an SSC Magnet Have Just Two Supports", SSC-N-267, November 25, 1986.

2. "Superconducting Super Collider Magnet System Requirements", Revision A, October 23, 1986.

3. J.H. Faupel and F.E. Fisher, "Engineering Design", John Wiley and Sons, New York, 1981.

4. R.J. Roark and W.C. Young, "Formulas for Stress and Strain", 5th edition, McGraw-Hill, New York, 1975 . 


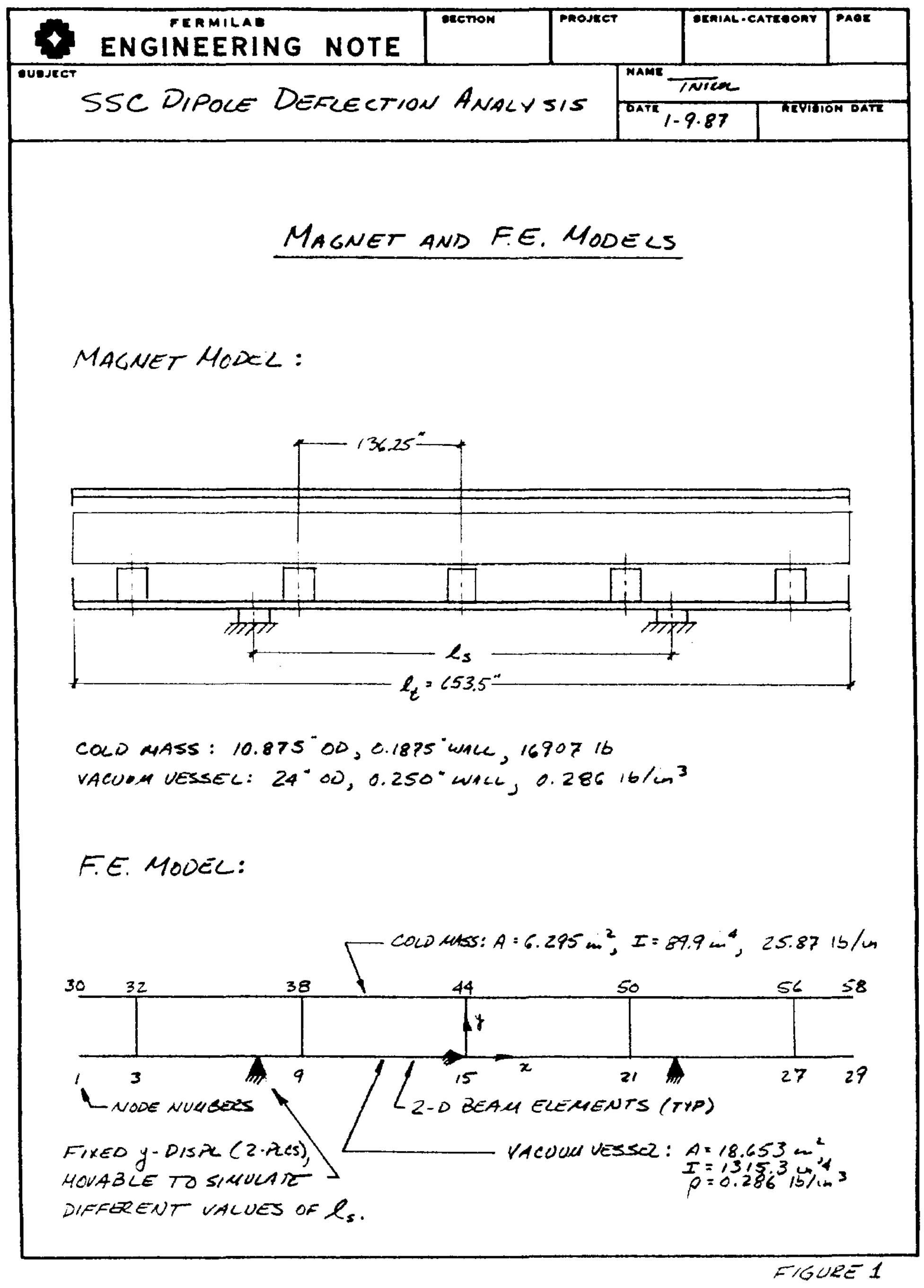




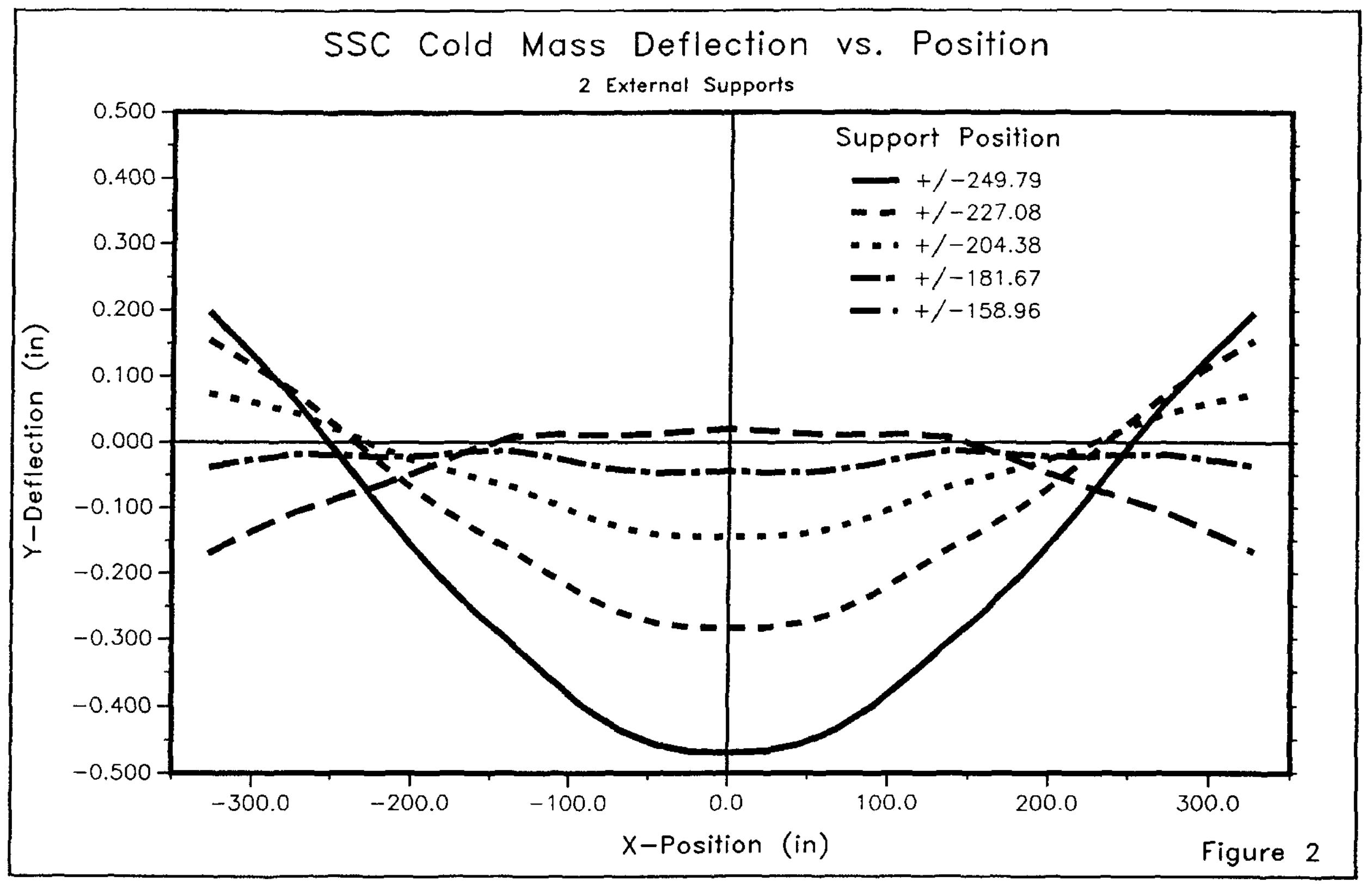




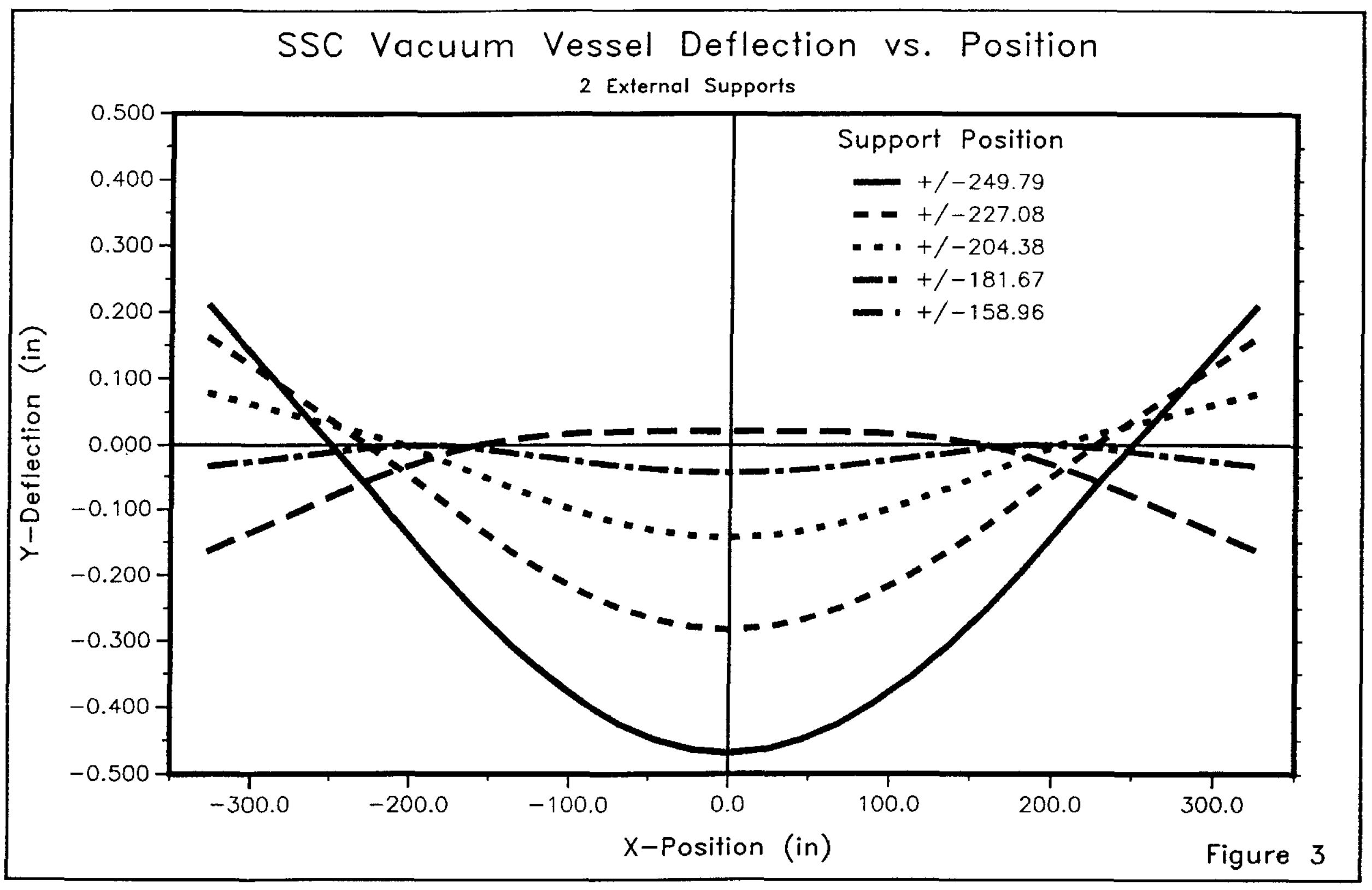




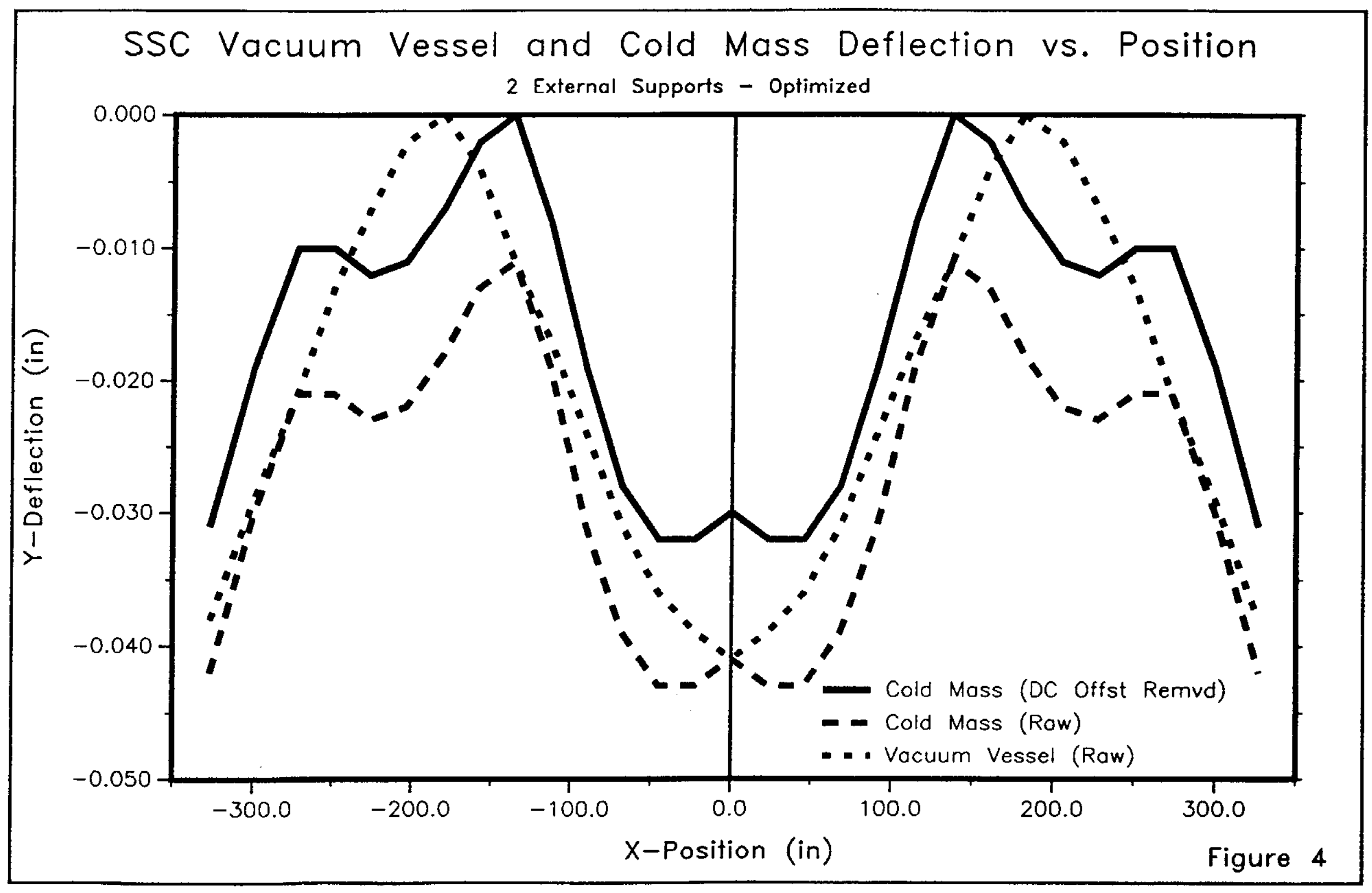




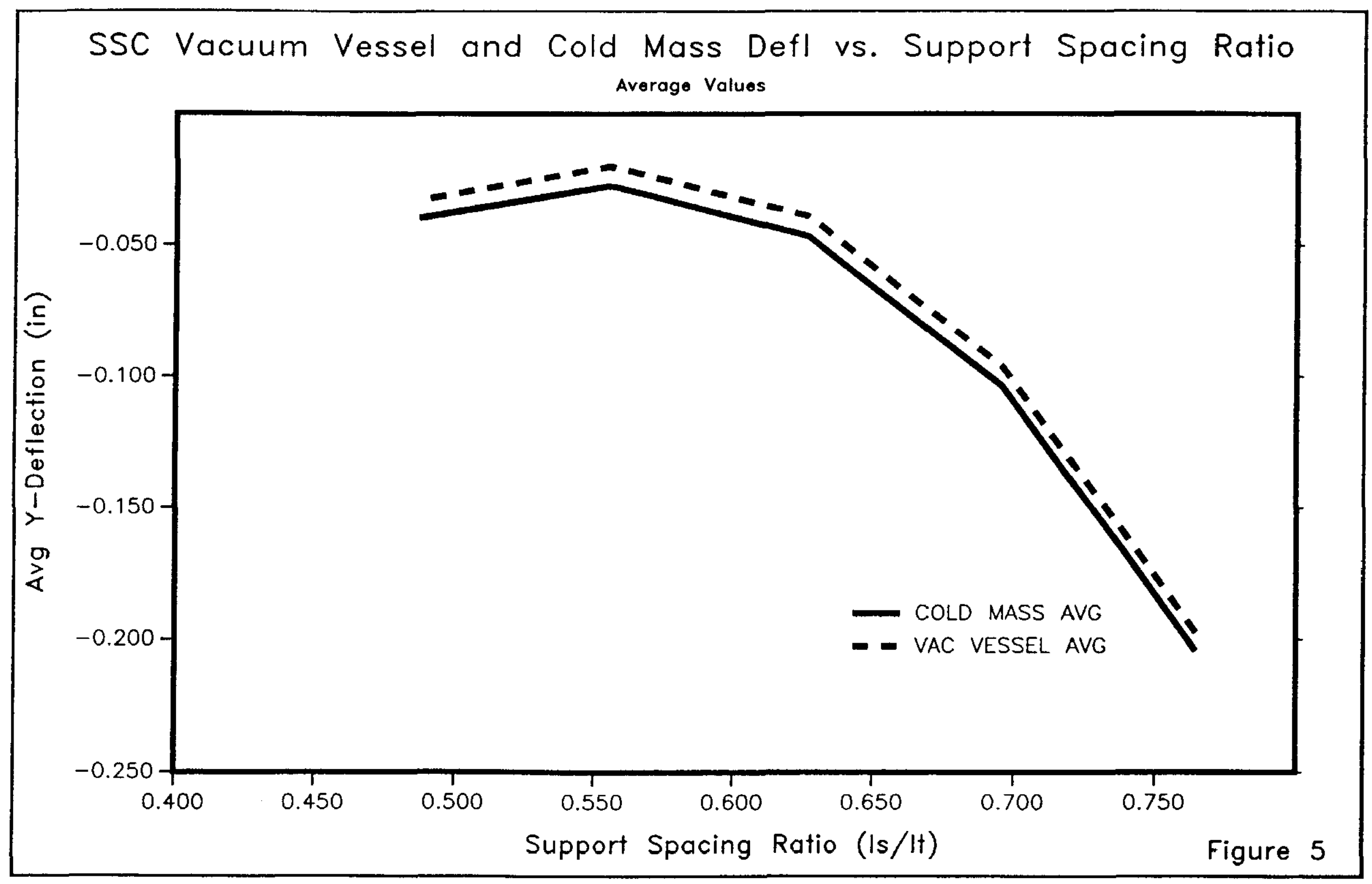




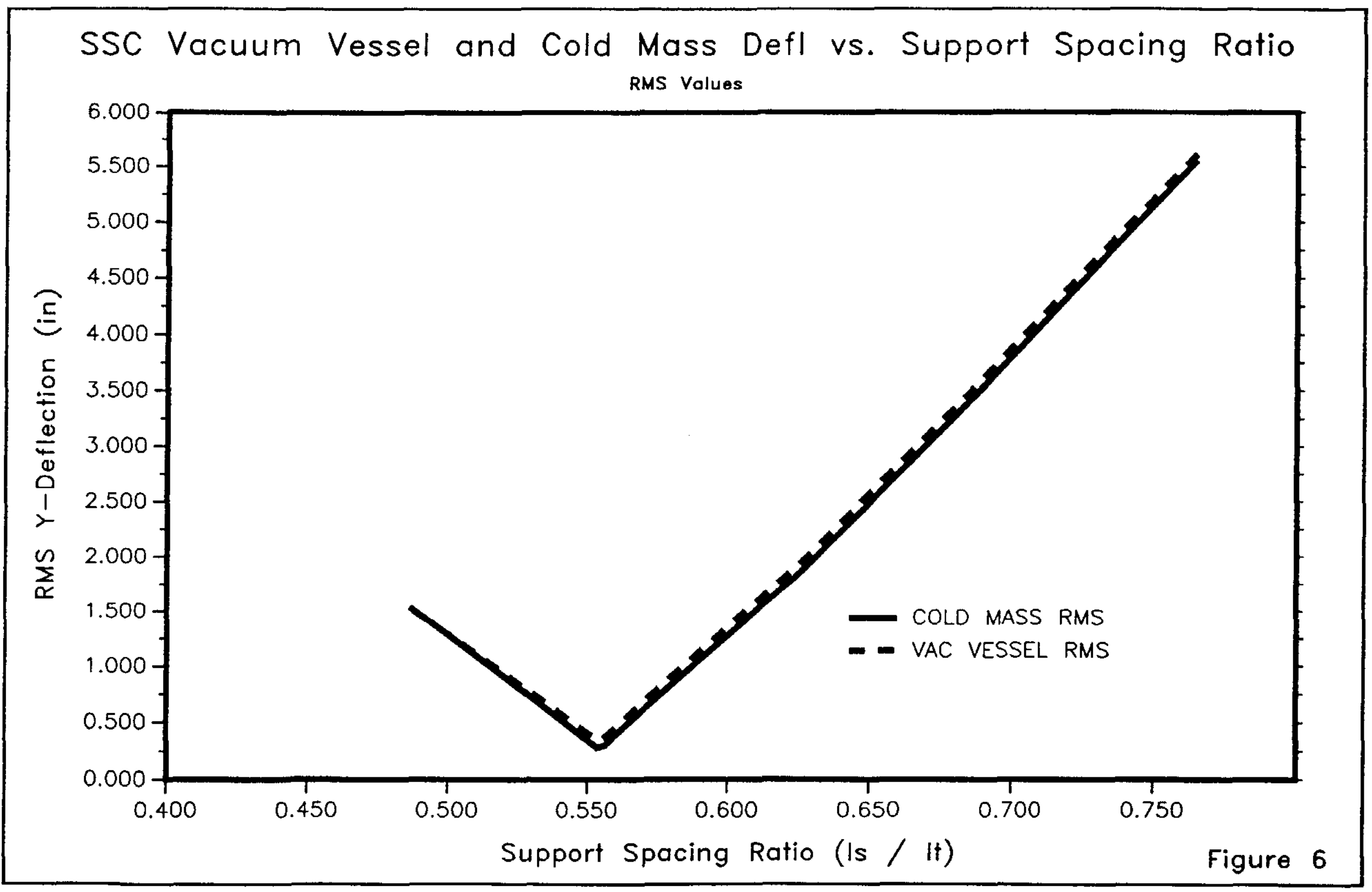




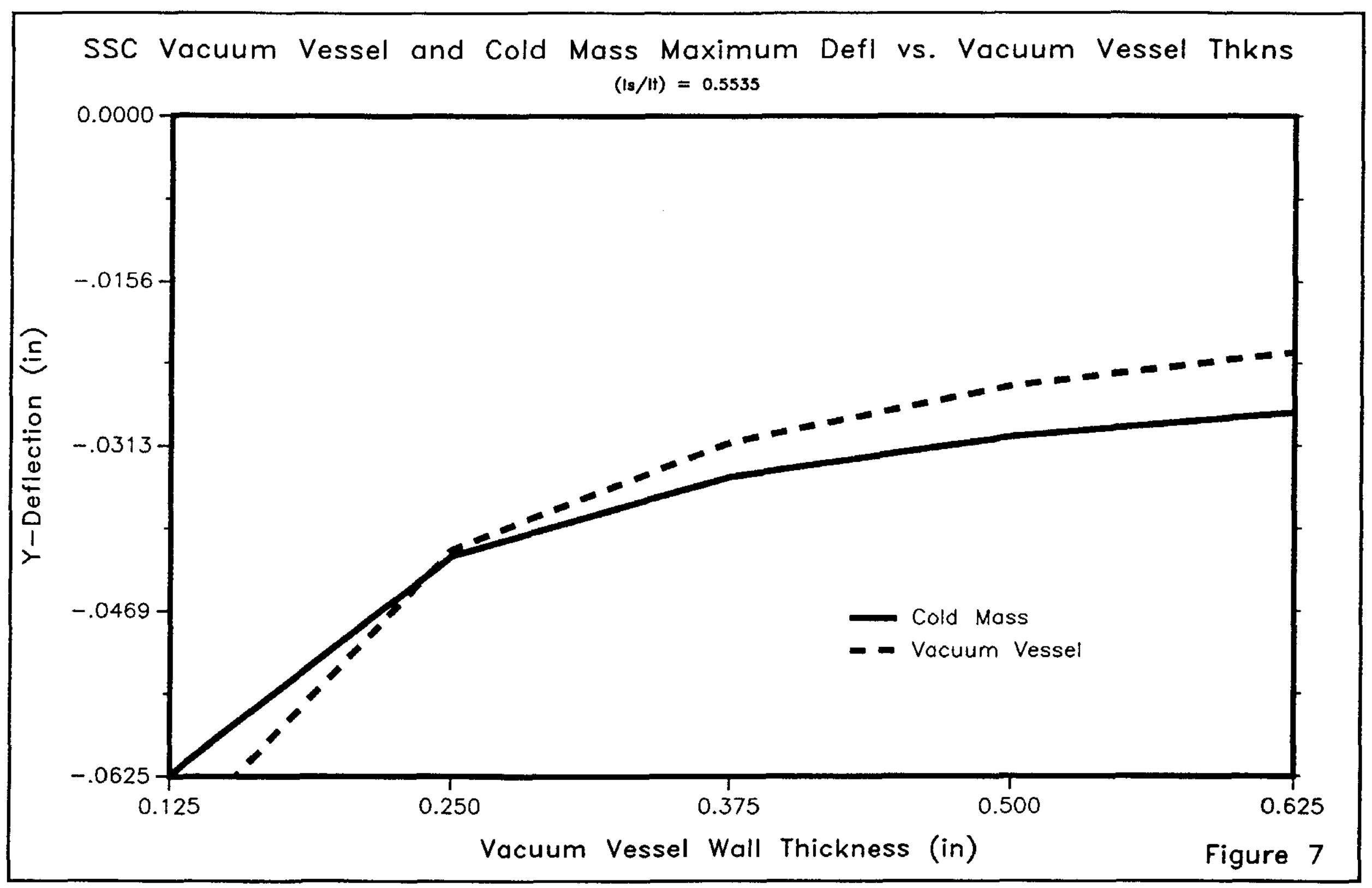




\section{Tablo 1}

Cold Mass and Vacuum Vessel Deflections vs. X-Position (supports positioned at $+/-180.86^{\prime \prime}$ )

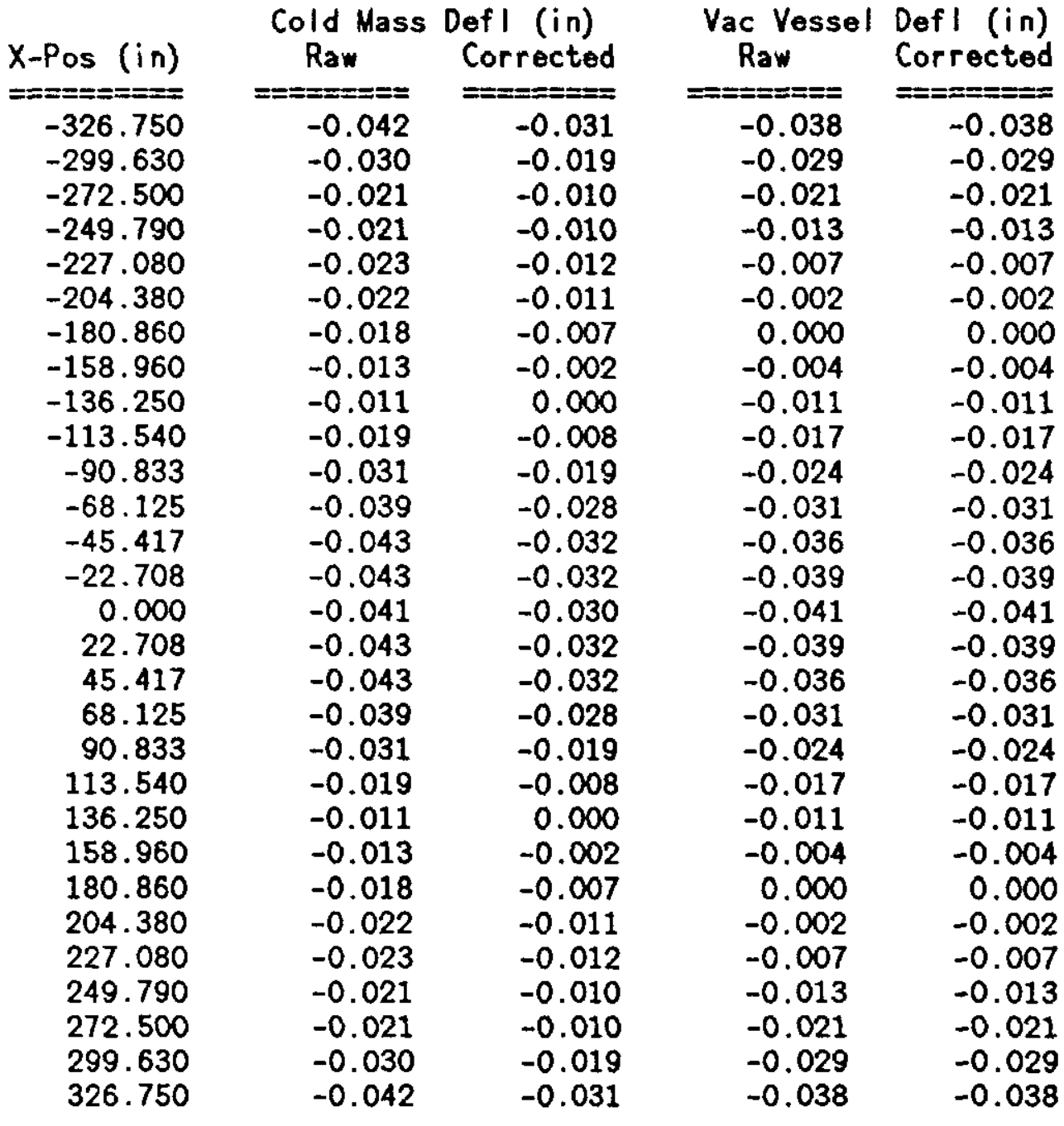

Raw Average (in) ........... Cold Mass: $\quad-0.028$

Vacuum Vessel: $\quad-0.020$

Corrected Average (in)....... Cold Mass: $\quad-0.016$

Vacuum Vessel: $\quad-0.020$

'Corrected' refers to the raw deflections less any 'DC' offset. 